\title{
Effects of local population structure in a reaction-diffusion model of a contact process on metapopulation networks
}

\author{
Angélica S. Mata, ${ }^{1}$ Silvio C. Ferreira, ${ }^{1}$ and Romualdo Pastor-Satorras ${ }^{2}$ \\ ${ }^{1}$ Departamento de Física, Universidade Federal de Viçosa, 36571-000, Viçosa-MG, Brazil \\ ${ }^{2}$ Departament de Física i Enginyeria Nuclear, Universitat Politècnica de Catalunya, Campus Nord B4, 08034 Barcelona, Spain
}

(Received 11 April 2013; revised manuscript received 21 May 2013; published 30 October 2013)

\begin{abstract}
We investigate the effects of local population structure in reaction-diffusion processes representing a contact process $(\mathrm{CP})$ on metapopulations represented as complex networks. Considering a model in which the nodes of a large scale network represent local populations defined in terms of a homogeneous graph, we show by means of extensive numerical simulations that the critical properties of the reaction-diffusion system are independent of the local population structure, even when this one is given by a ordered linear chain. This independence is confirmed by the perfect matching between numerical critical exponents and the results from a heterogeneous mean-field theory suited, in principle, to describe situations of local homogeneous mixing. The analysis of several variations of the reaction-diffusion process allows us to conclude the independence from population structure of the critical properties of CP-like models on metapopulations, and thus of the universality of the reaction-diffusion description of this kind of models.
\end{abstract}

DOI: 10.1103/PhysRevE.88.042820

PACS number(s): 89.75.Hc, 05.70.Jk, 05.10.Gg, 64.60.an

\section{INTRODUCTION}

The interplay between topology and dynamics rates among the most outstanding challenges in complex network theory [1]. Supported by a large amount of scientific evidence, it has become clear that dynamical processes running on top of a complex network can be heavily influenced by the topological properties of the substrate [2,3], especially when the network shows a strongly heterogeneous pattern of connections, as in the case of the so-called scale-free (SF) networks, with a degree distribution (probability that a vertex is connected to $k$ others) scaling as a power law $P(k) \sim k^{-\gamma}$ [4]. Initial research on particle interaction models in networks focused on fermionic models, in which every node of the network can be occupied by at most one particle. Within this framework, a number of remarkable results were developed, concerning the effects of large degree fluctuations in the behavior of processes both in and out of equilibrium [5-12].

Recently, a new theoretical framework has been proposed to study general dynamical processes on complex networks, based in the concept of reaction-diffusion processes on metapopulations [13-15]. Reaction-diffusion (RD) processes [16] are dynamical systems defined in terms of different kinds of particles or "species," which diffuse stochastically and interact among them according to a given set of reaction rules. On the other hand, metapopulation is a concept coined in ecology that refers to the structural organization of populations as discrete entities that interact via migration or gene flow [17]. Metapopulation ecology deals, among other subjects, with the local extinction and recolonization of spatially isolated patches, the evolutionary and genetic events involved in these processes, as well as the impact of human activity in the population dynamics [17].

In the context of network theory, considering RD processes on metapopulations allows a bosonic view, in which a node can host more than one particle, particle interactions take place inside the nodes of the network (thus representing the metapopulations), and particle transport (mediated by diffusion) takes place among nodes connected by edges. RD processes on metapopulations have been successfully applied to study different dynamics [14,18], but their most fruitful application is on epidemic spreading [19-22], where metapopulations provide a realistic description of the multiscale sociogeographical organization of people in countries, cities, neighborhoods, and so forth, where epidemics take place, and in which the transportation patterns are, in general, highly complex [23].

An important common factor in most previous models of $\mathrm{RD}$ processes on metapopulations is that, while they take into account the heterogeneity of the connectivity pattern of the set of populations (in terms of a complex network), they usually disregard the internal structure of the populations, taking a local homogeneous mixing approximation, equivalent to describe at mean-field level the reactions taking place inside each node. This approach raises the issue of the possible effects of the population inner structure in the behavior of the RD processes. In this paper, we tackle this problem by considering a metapopulation RD version of a contact process (CP) [24] on a network in which each vertex represents a structured population of fixed size given by a graph (see Fig. 1). By means of extensive numerical simulations, we show that the critical properties of the RD process are insensitive to the population structure, even in the extreme case of non-small-world [25] linear chains. This observation is confirmed by the perfect matching of numerical results with a heterogeneous mean-field solution $[2,3]$, that completely neglects population structure. The analysis of several variations of the model leads us to the extended conclusion that the critical properties of CP-like models on metapopulations depend exclusively on the topological structure of the metapopulation network.

We have organized the paper as follows: The reactiondiffusion process considered is defined in Sec. II, and the corresponding HMF theory is developed in Sec. III. The results of HMF theory are compared with quasistationary numerical simulations in Sec. IV. Finally, we draw our concluding remarks in Sec. V. 


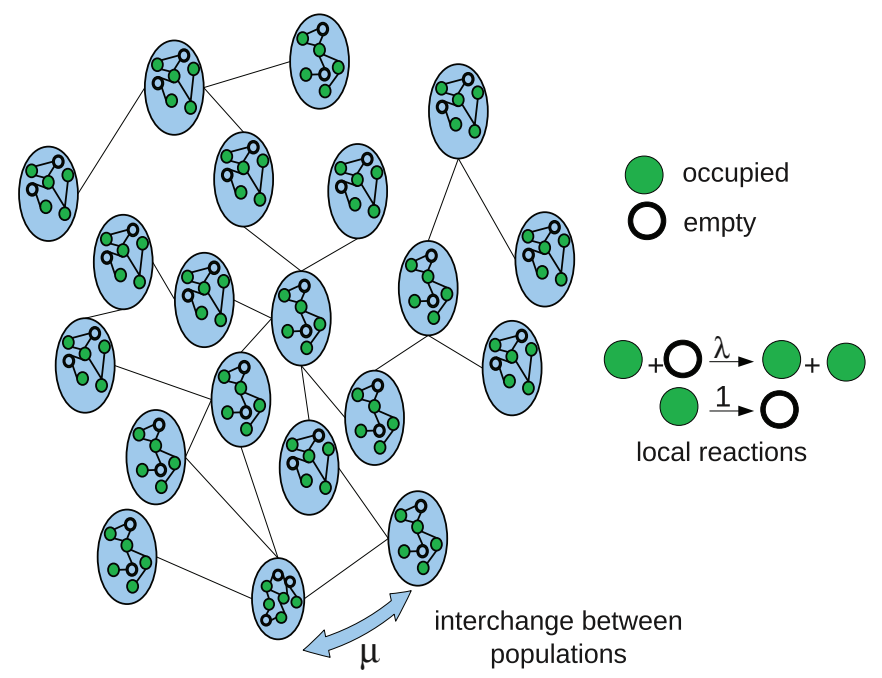

FIG. 1. (Color online) Reaction-diffusion process in heterogeneous metapopulations represented with a networked inner structure. Populations are depicted by large blue vertices, while the vertices inside each population are represented by circles. Particles in connected populations are exchanged at a rate $\mu$, create offspring in their nearest empty neighbors inside their respective populations at a rate $\lambda$, and become empty at a unitary rate.

\section{RD MODEL DEFINITION}

We consider a RD process on metapopulations representing a contact process characterized by two typical scales: local populations where reaction processes of creation and annihilation take place, and nonlocal (long-range) interconnections between populations (see Fig. 1). The system is defined in terms of $N$ populations (the metapopulation) of fixed size $L$ connected by nondirected edges, defining a nonlocal network with a degree distribution that we choose to have a SF form $P(k) \sim k^{-\gamma}$. Exchange of particles, via diffusion, takes place along the edges of this network. The populations themselves are considered as homogeneous graphs with average degree $z$. We have considered two forms for the local population network: a random regular network and a linear chain. The dynamics is defined as follows: Vertices inside populations may be in two states: either occupied or empty. An occupied site creates offsprings in all empty nearest neighbors (NNs) inside its population at a rate $\lambda$; occupied sites, on the other hand, annihilate spontaneously at rate 1 (this choice arbitrarily defines the dynamics time scale). Finally, particles migrate between two connected populations at a rate $\mu$. A migration event is performed by swapping a particle in a population and a vertex in a population connected by an edge. In our model, only occupied sites migrate.

The state devoid of particles represents an absorbing configuration from which the dynamics can not escape. We therefore expect that the system undergoes an absorbing-state phase transition [24], characterized by the order parameter $\rho$, defined as the average density of particles. The transition will take place at some critical point $\lambda_{c}(\mu)$, separating an active phase, with a constant average density of particles $(\rho>0)$, and the absorbing state with $\rho=0$.

\section{HMF THEORY}

The analytical study of the absorbing-state phase transition in this model is a complex task, particularly in the case of local population in the shape of a linear chain. We will therefore make the strong simplification of assuming homogeneous mixing inside populations. In this case, the problem is amenable to analytical solution by applying the heterogeneous mean-field (HMF) approximation [2,3]. Under these conditions, the evolution equation for the density $\rho_{k}$ of occupied sites in a population of degree $k$ takes the form

$$
\begin{aligned}
\frac{d \rho_{k}}{d t}= & -\rho_{k}+z \lambda \rho_{k}\left(1-\rho_{k}\right)+\mu k\left(1-\rho_{k}\right) \sum_{k^{\prime}} \frac{\rho_{k^{\prime}} P\left(k^{\prime} \mid k\right)}{k^{\prime}} \\
& -\rho_{k} \mu \sum_{k^{\prime}}\left(1-\rho_{k^{\prime}}\right) P\left(k^{\prime} \mid k\right)
\end{aligned}
$$

where the conditional probability $P\left(k^{\prime} \mid k\right)$ gives the probability that a node of degree $k$ is connected to a node of degree $k^{\prime}$ [26]. The first term of Eq. (1) represents spontaneous annihilation, while the second one represents a creation event in empty sites with $z$ NNs. Notice that these two terms assume homogeneous mixing. The third and fourth terms represent the incoming and outgoing diffusive flow contributions due to migration of occupied sites. The factor $1 / k^{\prime}$ in the third term reckons particles migrating to neighbor populations with equal chance. A stability analysis of Eq. (1) around the trivial empty state fixed point $\rho_{k}=0$ yields the linearized equation

$$
\frac{d \rho_{k}}{d t}=\sum_{k^{\prime}} L_{k k^{\prime}} \rho_{k^{\prime}}(t)+\mathcal{O}\left(\rho_{k}^{2}\right),
$$

where the Jacobian matrix is $L_{k k^{\prime}}=-(1-z \lambda+\mu) \delta_{k k^{\prime}}+$ $\mu k P\left(k^{\prime} \mid k\right) / k^{\prime}, \delta_{k k^{\prime}}$ being the Kronecker delta symbol. The active phase is obtained when the trivial fixed point becomes unstable, which happens if the largest eigenvalue of the Jacobian is positive. Obviously, the largest eigenvalue of $L_{k k^{\prime}}$ can be written as $\ell_{m}=-(1-z \lambda+\mu)+\mu c_{m}$, where $c_{m}$ is the largest eigenvalue of $C_{k k^{\prime}}=k P\left(k^{\prime} \mid k\right) / k^{\prime}$. It is easy to show that $v_{k}=k$ is an eigenvector of $C_{k k^{\prime}}$ with eigenvalue $c=1$. The matrix $C_{k k^{\prime}}$ is irreducible because the network is connected and, consequently, the Perron-Frobenius theorem [1] implies that $C_{k k^{\prime}}$ has a unique eigenvector with positive components that corresponds to the largest eigenvalue. Therefore, $c_{m}=$ $c=1$ and condition $\ell_{m}>0$ gives the critical point $\lambda_{c}=1 / z$.

To obtain information about other critical properties, we consider uncorrelated networks for which $P\left(k^{\prime} \mid k\right)=$ $k^{\prime} P\left(k^{\prime}\right) /\langle k\rangle$ [27], which leads to the equation

$$
\frac{d \rho_{k}}{d t}=z \Delta \rho_{k}-\lambda z \rho_{k}^{2}+\frac{\mu k \rho}{\langle k\rangle}\left(1-\rho_{k}\right)-\rho_{k} \mu\left(1-\frac{\Theta}{\langle k\rangle}\right),
$$

where $\Delta=\lambda-\lambda_{c}, \rho=\sum_{k} \rho_{k} P(k)$, and $\Theta=\sum_{k} k \rho_{k} P(k)$. From here, we can obtain the equation for the total particle density, which takes the form

$$
\frac{d \rho}{d t}=-\rho+z \lambda\left(\rho-\left\langle\rho_{k}^{2}\right\rangle\right)
$$

where the diffusion terms have canceled due to conservation of particles implied in this process. From this expression, we 
obtain in the steady state

$$
\left\langle\rho_{k}^{2}\right\rangle=\Delta \rho / \lambda
$$

where $\left\langle\rho_{k}^{2}\right\rangle=\sum_{k} P(k) \rho_{k}^{2}$.

A quasistatic approximation [28], assuming $\dot{\rho}_{k} \approx 0$ and isolating $\rho_{k}$ in Eq. (3), yields

$$
\rho_{k}=\frac{\varrho k}{1+\varrho k}+\frac{(\varrho k)^{2}}{(1+\varrho k)^{3}}+\mathcal{O}\left[(\varrho k)^{3}\right],
$$

where $\varrho=\rho /[\langle k\rangle(1-z \Delta / \mu)-\Theta]$, and we have performed an expansion up to order $\rho^{2}$, which should be valid very close to the transition. Inserting Eq. (6) into the definition of $\left\langle\rho_{k}^{2}\right\rangle$, replacing the summation by an integral for a normalized degree distribution $P(k)=A_{\gamma} k^{-\gamma}$ with $k=k_{0}, \ldots, k_{c}, A_{\gamma}=(\gamma-$ 1) $k_{0}^{\gamma-1}, k_{c} \gg k_{0}$, and $\gamma>2$, we obtain, in the limit $\varrho k_{c} \gg 1$, corresponding to a super-critical phase in a infinite system limit,

$$
\left\langle\rho_{k}^{2}\right\rangle= \begin{cases}\frac{(\gamma-1) \Gamma(3-\gamma) \Gamma(\gamma)}{2}\left(k_{0} \varrho\right)^{\gamma-1}, & 2<\gamma<3 \\ \frac{(\gamma-1)^{2}}{\gamma-3}\left(k_{0} \varrho\right)^{2}, & \gamma>3\end{cases}
$$

where $\Gamma(x)$ is the gamma function [29].

From Eqs. (5) and (7), and using that $\varrho \simeq \rho /\langle k\rangle$ very close to the critical point, we find $\rho \sim \Delta^{\beta}$, with a critical exponent $\beta=1 /(\gamma-2)$ if $2<\gamma<3$ and $\beta=1$ if $\gamma \geqslant 3$. At criticality, setting $\lambda=1 / z$, the density evolves as $d \rho / d t=-\left\langle\rho_{k}^{2}\right\rangle$. Using the asymptotic form of $\left\langle\rho_{k}^{2}\right\rangle \sim \rho^{b}$ given by Eq. (7), one obtains $\rho \sim t^{-\delta}$ with $\delta=\beta$. We can also show that close to the critical point the density approaches the asymptotic value as $\rho(t)=$ $\rho_{s}+$ const $\times \exp (-t / \tau)$ where the characteristic time diverges as $\tau=(z b \Delta)^{-1} \sim \Delta^{-v_{\|}}$with an exponent $v_{\|}=1$.

Interestingly, these critical exponents coincide with the HMF solution for the contact process in uncorrelated networks [30,31]. This fact suggests an extension of the contact process approach to compute the finite-size scaling (FSS) behavior of our model. Indeed, applying the strategy proposed in Ref. [30], in which the motion equation is mapped in a one-step process, we find (see Appendix A for details) that the stationary density of occupied vertices $\bar{\rho}$ and the characteristic time $\tau$ display an anomalous size dependence at the transition given by

$$
\bar{\rho} \sim(\Omega g)^{-1 / 2} \quad \text { and } \quad \tau \sim(\Omega / g)^{1 / 2},
$$

where $g=\frac{\left\langle k^{2}\right\rangle}{\langle k\rangle^{2}}$ and $\Omega=N L$. Since $g$ depends only of $N$ through the network cutoff $k_{c} \sim N^{1 / \omega}$ [32], we have that $\bar{\rho} \sim$ $L^{-1 / 2} N^{-\hat{v}}$ and $\tau \sim L^{1 / 2} N^{\hat{\alpha}}$ where

$$
\hat{v}=\frac{1}{2}+\max \left(\frac{3-\gamma}{2 \omega}, 0\right), \quad \hat{\alpha}=\frac{1}{2}-\max \left(\frac{\gamma-3}{2 \omega}, 0\right) \text {. }
$$

\section{QUASISTATIONARY SIMULATIONS}

\section{A. Methods}

In order to check the predictions of the HMF theory, we performed extensive simulations of the RD process in heterogeneous metapopulations. The networks connecting populations were simulated using the uncorrelated configuration model (UCM) [33], that warrants the absence of degree correlation. Letting $(i, j)$ represent the vertex $j$ inside

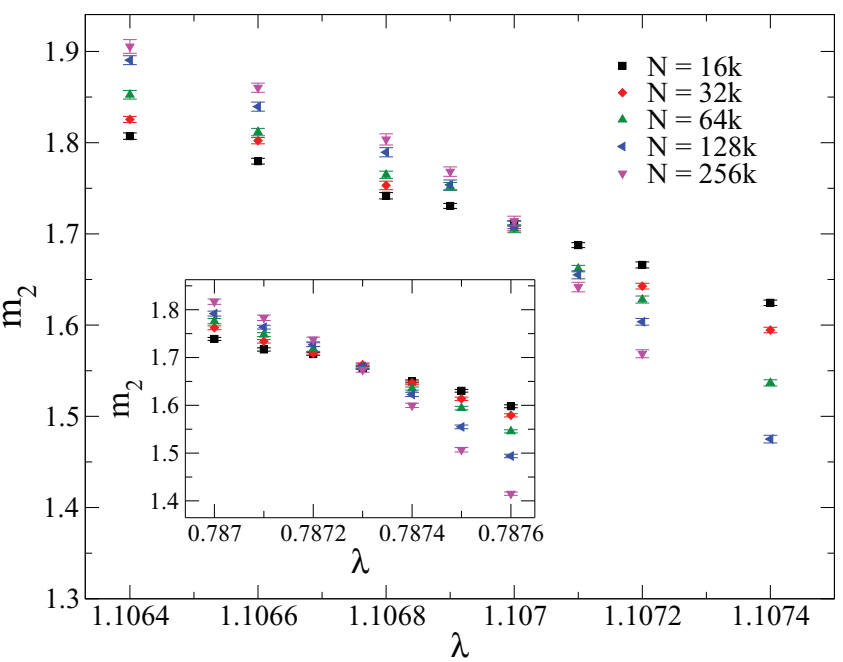

FIG. 2. (Color online) Moment ratios for the reaction-diffusion processes on metapopulations connected by UCM networks with minimum degree $k_{0}=6$ and cutoff $k_{c}=N^{1 / 2}$. In the main plot, the migration rate is $\mu=0.10$, degree exponent is $\gamma=2.75$, and the local population structure of the populations are RRNs with size $L=250$ and $z=2$. In the inset, the parameters $\mu=0.50, \gamma=2.25$ and regular chains of size $L=250$ and $z=2$ are used.

the population $i$, the computer algorithm is implemented as follows: At each time step, an occupied vertex $(i, j)$ is chosen and the time increased by $\Delta t=1 /[n(1+\lambda+\mu)]$, where $n$ is the number of particles at time $t$. With probability $p=$ $1 /(1+\lambda+\mu)$, the vertex becomes vacant. With probability $q=\lambda /(1+\lambda+\mu)$, all empty nearest neighbors of $j$ inside population $i$ are occupied. Finally, the migration to a randomly chosen vertex $\left(i^{\prime}, j^{\prime}\right)$ belonging to a neighbor population occurs with probability $r=1-p-q$.

We used a quasistationary (QS) simulation method where, every time the system visits the absorbing state, the absorbing configuration is replaced by a state randomly taken from the system history $[34,35]$. To implement the method, a list containing $M=100$ configurations is stored and constantly updated. The updating is done by randomly picking up a stored configuration and replacing it by the current one with probability $p_{r} \Delta t$. We fixed $p_{r} \simeq 10^{-4}-10^{-3}$ (the larger $N$, the smaller $p_{r}$ ). After a relaxation time $t_{r}=10^{6}$, the QS averages are computed over times up to $t_{a v}=2 \times 10^{7}$. Ensembles of 50 network configurations were used for statistical averages.

The method used to determine critical points in simulations is based in the idea of the size independence at criticality of the moment ratio $m_{2}=\left\langle\rho^{2}\right\rangle /\langle\rho\rangle^{2}$ [35-37]: by plotting the ratio $m_{2}$ as a function of $\lambda$ for different values of the network size $N$, the critical point will be given by the intersection of the plots for all network sizes. Figure 2 shows typical curves used to determine the critical point using the moment ratio technique.

\section{B. Numerical results}

First, we have considered the case of local populations with a random structure, represented by a random regular network (RRN) [38] with fixed average degree $z=2$ and random connections excluding self- and multiple edges. Table I shows the respective critical points for different migration 
TABLE I. Critical points for the RD process on populations connected by UCM networks with minimum degree $k_{0}=6$, cutoff $k_{c}=N^{1 / 2}$, and degree exponent $\gamma$. The population structure consists in either a RRN or a linear chain with $L=250$ vertices and $z=2$. The thresholds in a pair approximation $\lambda_{c}^{\mathrm{PA}}=(1+\mu) /(1+2 \mu)$ are also shown. Numbers in parentheses represent uncertainties in the last digit.

\begin{tabular}{|c|c|c|c|c|c|c|c|c|}
\hline & \multicolumn{4}{|c|}{ Random regular networks } & \multicolumn{4}{|c|}{ Linear chain } \\
\hline & $\mu=0.05$ & $\mu=0.10$ & $\mu=0.50$ & $\mu=0.90$ & $\mu=0.05$ & $\mu=0.10$ & $\mu=0.50$ & $\mu=0.90$ \\
\hline$\gamma=2.25$ & $1.2361(1)$ & $1.10585(5)$ & $0.78995(5)$ & $0.68680(5)$ & $1.23315(5)$ & $1.1031(1)$ & $0.7873(1)$ & $0.69445(5)$ \\
\hline$\gamma=2.75$ & $1.23705(5)$ & $1.1070(1)$ & $0.79145(5)$ & $0.69820(5)$ & $1.2336(1)$ & $1.1034(1)$ & $0.78740(5)$ & $0.69455(5)$ \\
\hline$\gamma=3.50$ & $1.2380(1)$ & $1.10820(5)$ & $0.7930(1)$ & $0.69970(5)$ & $1.2340(5)$ & $1.1038(1)$ & $0.7876(1)$ & $0.69465(5)$ \\
\hline PA & 0.954 & 0.916 & 0.75 & 0.678 & 0.954 & 0.916 & 0.75 & 0.678 \\
\hline
\end{tabular}

rates and degree exponents. We observe that the critical points are almost independent of the heterogeneity of the population network, expressed by the degree exponent $\gamma$, in agreement with the results obtained for the contact process in a fermionic approach [36]. On the other hand, we observe that the critical point decreases for increasing migration rate, at odds with the constant $\mathrm{HMF}$ prediction $\lambda_{c}=1 / z$. This observation is, however, reasonable since migration facilitates activity spreading to nonactive regions, and can be accounted for by means of a simple homogeneous pair approximation $[36,37,39]$. Within this approach (see Appendix B), we obtain a new threshold $\lambda_{c}^{\mathrm{PA}}(\mu)=(1+\mu) /(z-1+\mu z)$ that is closer to simulation results than the HMF prediction.

The great trump of the HMF theory in absorbing state phase transitions is to predict the correct FSS critical exponents even for quenched networks [36,37], where neglecting dynamical correlations implies an approximation. Figure 3 shows an example of FSS for critical density $\bar{\rho}$ and migration rate $\mu=0.1$. Similar plots are obtained for the characteristic time $\tau$, as illustrated in Fig. 4. The power law regressions in plots of $\bar{\rho}$ and $\tau$ versus $N$ yield exponents varying with network
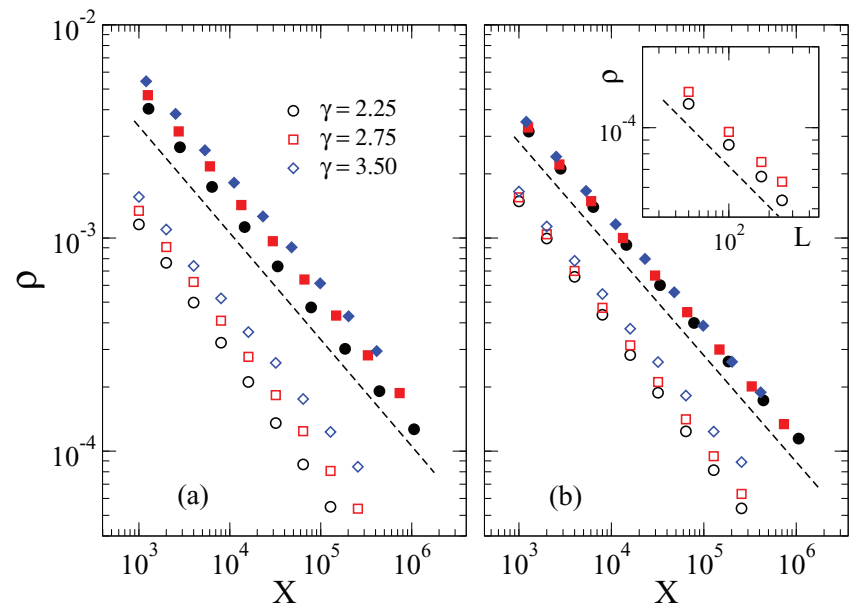

FIG. 3. (Color online) Finite size scaling of the critical density for distinct structured local populations. Results for (a) RRN and (b) linear chain are shown. The migration rate is $\mu=0.1$ and the remaining fixed parameters are as in Table I. The curves $\bar{\rho}$ vs $N$ are represented by open symbols while curves $\rho$ vs $g N, g=\left\langle k^{2}\right\rangle /\langle k\rangle^{2}$, are represented by the filled ones. Inset in (b) shows the critical density against $L$ for $N=256000$ and $\gamma=2.75$ for RRN (circles) and linear chains (squares). Filled symbols were shifted to improve visibility. Dashed lines represent the power law $X^{-1 / 2}$. heterogeneity but independent of the exchange rate. The exponents apparently differ from HMF predictions given by Eq. (9) as shown in Table II. However, the $\left\langle k^{2}\right\rangle$ and $\langle k\rangle$ factors in Eq. (8) introduce strong corrections to scaling [31,35,36], which can be explicitly taken into account by performing power law regressions of $\bar{\rho}$ versus $g N$ and $\tau$ versus $N / g$. In this way, the HMF scaling law $\bar{\rho} \sim(g N)^{-S_{v}}$ with $S_{v}=\frac{1}{2}$ is recovered in all simulations, as shown in Fig. 3 and Table II for $\mu=0.1$. The scaling $\tau \sim(N / g)^{S_{\alpha}}$ with $S_{\alpha}=\frac{1}{2}$ is also very well verified, as one can see in Fig. 4. For fixed $N$, the scaling laws $\bar{\rho} \sim L^{-1 / 2}$ and $\tau \sim L^{1 / 2}$ are also confirmed in simulations as shown in the inset of Fig. 3. However, the critical points slightly increase for smaller population sizes (from $\lambda_{c}=1.1070$ for $L=250$ to $\lambda_{c}=1.1105$ for $L=100$ ).

Turning now to the more interesting case of a population with a regularly ordered internal structure, we consider the case of a linear chain with coordination number $z=2$. Table I shows the critical points numerically computed for different migration rates. The critical points are practically identical to those found for RRNs. On the other hand, Figs. 3 and 4 show a surprisingly very good quantitative agreement between FSS critical exponents of the numerical simulations and the

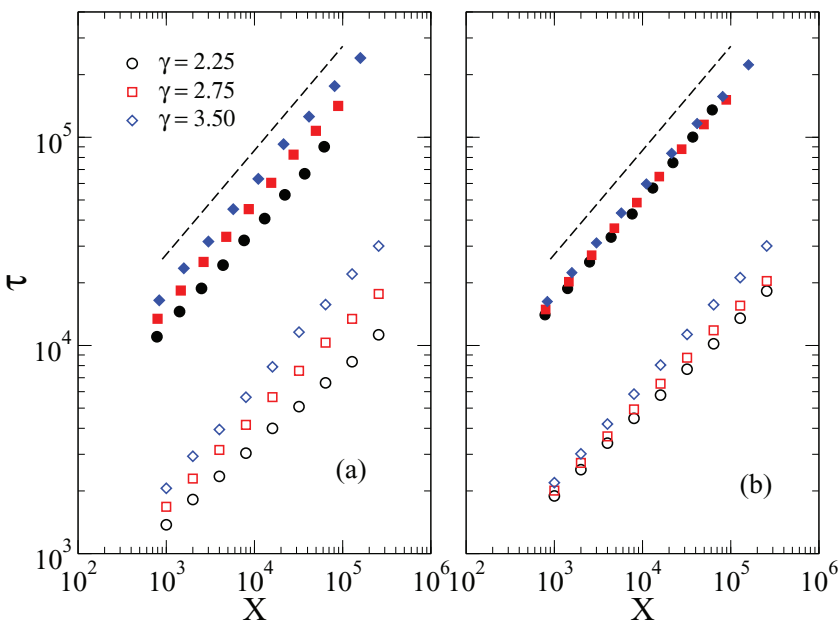

FIG. 4. (Color online) Finite size scaling of the critical characteristic time for distinct structured local populations. Results for (a) RRN and (b) linear chain are shown. The fixed parameters are as in Fig. 3. The curves $\tau$ vs $N$ are represented by open symbols while curves $\tau$ vs $N / g, g=\left\langle k^{2}\right\rangle /\langle k\rangle^{2}$ are represented by the filled ones. Filled symbols were shifted to improve visibility. Dashed lines represent the power law $X^{1 / 2}$. 
TABLE II. Critical exponents in the FSS of the quasistationary density and characteristic time: $\bar{\rho} \sim N^{-\hat{\nu}}, \bar{\rho} \sim(g N)^{-S_{\nu}}, \tau \sim N^{\hat{\alpha}}$, and $\tau \sim(N / g)^{S_{\alpha}}$. The HMF results for $\hat{v}$ and $\hat{\alpha}$ are also indicated, while the exponents $S_{v}=S_{\alpha}=\frac{1}{2}$ are independent of the degree exponent. The size of each population is $L=250$ and the migration rate is $\mu=0.1$.

\begin{tabular}{|c|c|c|c|c|c|c|c|c|c|c|}
\hline & \multicolumn{4}{|c|}{ RRN } & \multicolumn{4}{|c|}{ Chain } & \multicolumn{2}{|c|}{ HMF } \\
\hline & $\hat{v}$ & $S_{v}$ & $\hat{\alpha}$ & $S_{\alpha}$ & $\hat{v}$ & $S_{v}$ & $\hat{\alpha}$ & $S_{\alpha}$ & $\hat{v}$ & $\hat{\alpha}$ \\
\hline$\gamma=2.25$ & $0.63(2)$ & $0.51(2)$ & $0.37(2)$ & $0.48(2)$ & $0.60(2)$ & $0.49(2)$ & $0.40(3)$ & $0.51(2)$ & 0.69 & 0.31 \\
\hline$\gamma=2.75$ & $0.58(1)$ & $0.49(1)$ & $0.43(3)$ & $0.52(3)$ & $0.58(2)$ & $0.50(2)$ & $0.42(2)$ & $0.49(2)$ & 0.56 & 0.44 \\
\hline$\gamma=3.50$ & $0.52(2)$ & $0.50(1)$ & $0.49(2)$ & $0.51(2)$ & $0.53(2)$ & $0.50(1)$ & $0.47(3)$ & $0.50(3)$ & $1 / 2$ & $1 / 2$ \\
\hline
\end{tabular}

prediction of the HMF theory (see Table II). Results for the other values of $\mu$ yield the same critical exponent inside error bars.

The high accuracy of HMF to determine critical exponents for local populations composed of regular chains is somehow surprising since the structure of the chains strongly violates the hypothesis of homogeneous mixing used in the mean-field approach. However, we can see that the mixing is indirectly caused by the random movements through populations mediated by diffusion. This effect is nevertheless not just due to the random choice of the position inside the neighbor population. We have modified the model (variant 1) by using a deterministic migration rule in the choice of the new position inside a neighbor chain $\left(j^{\prime} \equiv j\right.$ in the model implementation previously described). This rule mimics, in a very simple fashion, the fact that individuals use to visit places regularly. This modification does not change the HMF theory. Numerically, simulations result in a small increase of the threshold (from $\lambda_{c} \approx 1.103$ to 1.114 for $\mu=0.1$ ) due to the reduction of the spreading power; critical exponents, however, are not altered. Simulations confirm the theoretical assertion (see Fig. 5).

The model is also robust to other alterations of the dynamical rules. For example, we have considered a modification (variant 2) consisting in replacing the exchange by the creation
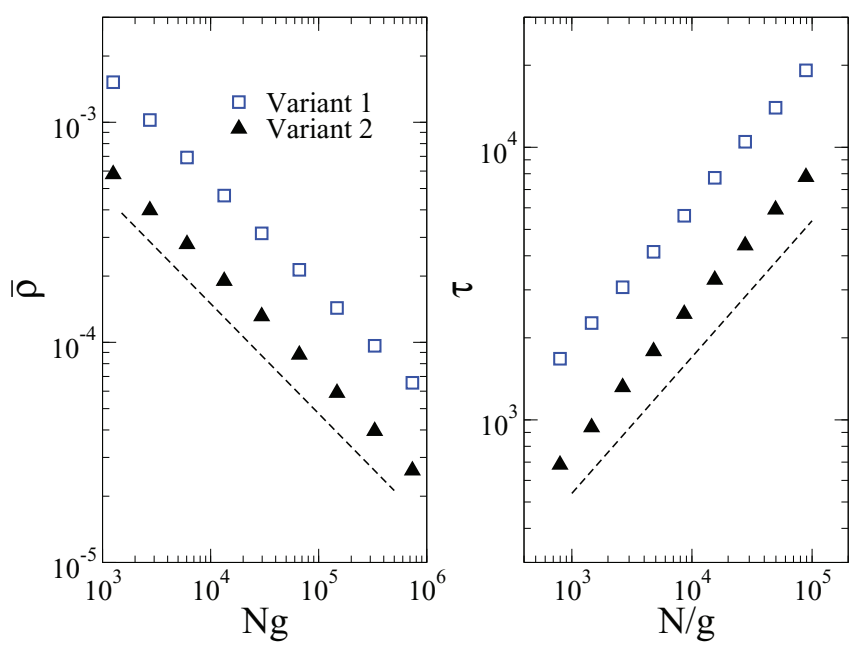

FIG. 5. (Color online) Finite size scaling of the critical density $\bar{\rho}$ and characteristic time $\tau$ in the variants of the RD process. In both variants, the structured local populations are represented by chains with $z=2$ nearest neighbors. The migration rate is $\mu=0.1$ and the degree exponent is $\gamma=2.75$. Power laws $(N g)^{-1 / 2}$ and $(N / g)^{1 / 2}$ are shown for comparison. of a new particle in a neighbor population at rate $\mu$. At the HMF level, the last term of Eq. (1) vanishes, the Jacobian matrix becomes $L_{k k^{\prime}}=-(1-z \lambda) \delta_{k k^{\prime}}+\mu C_{k k^{\prime}}$, and the critical $\lambda_{c}=$ $(1-\mu) / z$ is directly found, indicating that activity survives for any $\lambda$ if $\mu>1$. Simulations confirm the HMF predictions: the critical point is reduced (to $\lambda_{c} \approx 0.975$ for $\mu=0.1$ ), but critical exponents remain unchanged. Figure 5 shows the finite size scaling for critical density $\bar{\rho}$ and characteristic time $\tau$ for both variants.

\section{CONCLUSIONS}

In summary, we have investigated the effects of structured populations in reaction-diffusion (RD) processes representing a contact process (CP) on metapopulations modeled as complex networks. Extensive numerical simulations on a variety of model modifications show that the critical behavior of the RD processes is essentially independent of the local population structure. The critical exponents observed are well accounted for by a heterogeneous mean-field theory (HMF), and depend only on the topological properties of the metapopulation network; HMF theory fails to reproduce nonuniversal properties such as the critical point, which can be, however, recovered approximately by using a homogeneous pair approximation. The agreement between simulations and HMF theory is the more surprising for local populations with the form of a linear chain, violating even the smallworld property [25]. The results presented here represent compelling evidence of the universality of the metapopulation RD framework to study the critical properties of CP-like models on networks, and moreover indicate that an a priori strong oversimplification such as neglecting the local structure is indeed valid, which implies that a simplified setting with local homogeneous mixing is enough to reproduce processes in realistic settings.

\section{ACKNOWLEDGMENTS}

This project was supported by Brazilian agencies CNPq, FAPEMIG, and CAPES. R.P.-S. acknowledges financial support from the Spanish MEC, under Project No. FIS201021781-C02-01; the Junta de Andalucía, under Project No. P09-FQM4682; and ICREA Academia, funded by the Generalitat de Catalunya.

\section{APPENDIX A: FINITE SIZE SCALING THEORY}

A mean-field theory for the finite size scaling at criticality of the RD process under consideration can be obtained using 
the approach proposed in Ref. [30], in which the motion equation for $\rho$ is mapped in a one-step process. To obtain a self-consistent equation for $\rho$, we explicitly compute $\left\langle\rho_{k}^{2}\right\rangle$ by squaring Eq. (6) and keeping terms up to order $\mathcal{O}\left(\rho^{2}\right)$. The result for the stationary state is $\left\langle\rho_{k}^{2}\right\rangle \simeq \rho^{2} g$, where $g=$ $\left\langle k^{2}\right\rangle /\langle k\rangle^{2}$. In the limit of very low densities, we can substitute $\left\langle\rho_{k}^{2}\right\rangle$ into Eq. (4) to obtain the mean-field equation

$$
\frac{d \rho}{d t} \simeq-\rho+z \lambda \rho[1-\rho g] .
$$

The first term represents an annihilation process $n \rightarrow n-1$ while the second one represents a creation process $n \rightarrow n+1$. According to Ref. [30], the one-step process corresponding to Eq. (A1) is defined by the transition rates

$$
\begin{aligned}
& W(n-1, n)=n, \\
& W(n+1, n)=\lambda z n[1-\rho g],
\end{aligned}
$$

where $W(n, m)$ represents the transitions from a state with $m$ occupied sites to another state with $n$ occupied sites. The master equation for a standard one-step process is [16]

$$
\dot{P}_{n}=\sum_{m} W(n, m) P_{m}(t)-\sum_{m} W(m, n) P_{n}(t) .
$$

Substituting Eq. (A2), we find

$$
\dot{P}_{n}=(n+1) P_{n+1}+u_{n-1} P_{n-1}-\left(n+u_{n}\right) P_{n}
$$

with $u_{n}=\lambda n(1-\rho g)$. This equation was investigated in Ref. [35] within the QS analysis of the contact process in annealed scale-free networks. Since the solutions of Eq. (A4) have already been exhaustively investigated, we just report the results from Ref. [35]. The critical QS distribution of occupied vertices for large systems has the form

$$
\bar{P}_{n}=\frac{1}{\sqrt{\Omega / g}} f\left(\frac{n}{\sqrt{\Omega / g}}\right),
$$

where $f(x)$ is a scaling function and $\Omega=N L$ is the total number of vertices of the metapopulation. It directly follows from Eq. (A5) that the critical QS density scales as

$$
\bar{\rho} \equiv \frac{1}{\Omega} \sum_{n} n \bar{P}_{n} \sim(g \Omega)^{-1 / 2}
$$

and the characteristic time scales as

$$
\tau \equiv \frac{1}{\bar{P}_{1}} \sim\left(\frac{\Omega}{g}\right)^{1 / 2}
$$

For asymptotically large systems with a power law distribution $P(k) \sim k^{-\gamma}$, we have $g \sim k_{c}^{3-\gamma}$ for $\gamma<3$ and $g \sim$ const for $\gamma>3$ [31]. Since $g$ depends only on $N$, the scaling laws $\bar{\rho} \sim$ $L^{-1 / 2} N^{-\hat{v}}$ and $\tau \sim L^{1 / 2} N^{\hat{\alpha}}$ ensue in this way, with critical exponents $\hat{v}$ and $\hat{\alpha}$ as given by Eq. (9).

\section{APPENDIX B: HOMOGENEOUS PAIR APPROXIMATION}

We can develop a homogeneous pair approximation for our RD process following the procedure described in Refs. [37,40]. To do so, let us define the probability $Q\left(\sigma \sigma^{\prime}\right)$ that a pair of neighbor sites in a homogeneous population are in the states $\left(\sigma \sigma^{\prime}\right)$, where $\sigma=0$ (1) stands for an empty (occupied) node. The homogeneity inside populations implies $Q\left(\sigma \sigma^{\prime}\right)=$ $Q\left(\sigma^{\prime} \sigma\right)$. Let us define also the probabilities $\rho=Q(1)$, $\phi=Q(01), \psi=Q(11)$, and $\omega=Q(00)$. The normalization condition $2 \phi+\psi+\omega=1$ and the relation $\rho=\phi+\psi$ apply. The motion equation for $\rho$ and $\psi$ are

$$
\frac{d \rho}{d t}=-\rho+z \lambda \phi
$$

and

$$
\begin{aligned}
\frac{d \psi}{d t}= & -2 \psi+2 \lambda \phi+2 \lambda(z-1) Q(101) \\
& +2 \phi \alpha \rho-2 \psi \alpha(1-\rho) .
\end{aligned}
$$

The meaning of the different terms is analogous to those given in the paper. Equation (B1) can be directly obtained from Eq. (1) by considering the homogeneous approximation $\rho_{k}=$ $\rho$ and $k=\langle k\rangle$ and replacing $\rho(1-\rho)$ by $\phi$ in the second term. In a homogeneous pair approximation, the probability of a microscopic configuration is factorized as [41]

$$
Q\left(\sigma_{i} \sigma_{j} \sigma_{l}\right) \approx \frac{Q\left(\sigma_{i} \sigma_{j}\right) Q\left(\sigma_{j} \sigma_{l}\right)}{Q\left(\sigma_{j}\right)} .
$$

Therefore, Eq. (B2) turns to

$$
\frac{d \psi}{d t}=-2 \psi+2 \lambda \phi+\frac{2(z-1) \lambda \phi^{2}}{(1-\rho)}+2 \mu \phi \rho-2 \mu \psi(1-\rho) .
$$

Taking finally the stationary solutions of Eqs. (B4) and (B1), and using $\rho=\phi+\psi$, we find the critical point

$$
\lambda_{c}^{\mathrm{PA}}=\frac{1+\mu}{z-1+\mu z} .
$$

[1] M. Newman, Networks: An Introduction (Oxford University Press, Oxford, UK, 2010).

[2] A. Barrat, M. Barthélemy, and A. Vespignani, Dynamical Processes on Complex Networks (Cambridge University Press, Cambridge, UK, 2008).

[3] S. N. Dorogovtsev, A. V. Goltsev, and J. F. F. Mendes, Rev. Mod. Phys. 80, 1275 (2008).

[4] A.-L. Barabási and R. Albert, Science 286, 509 (1999).

[5] M. Leone, A. Vázquez, A. Vespignani, and R. Zecchina, Eur. Phys. J. B 28, 191 (2002).
[6] S. N. Dorogovtsev, A. V. Goltsev, and J. F. F. Mendes, Phys. Rev. E 66, 016104 (2002).

[7] R. Cohen, K. Erez, D. ben-Avraham, and S. Havlin, Phys. Rev. Lett. 85, 4626 (2000).

[8] D. S. Callaway, M. E. J. Newman, S. H. Strogatz, and D. J. Watts, Phys. Rev. Lett. 85, 5468 (2000).

[9] R. Pastor-Satorras and A. Vespignani, Phys. Rev. Lett. 86, 3200 (2001).

[10] M. E. J. Newman, Phys. Rev. E 66, 016128 (2002).

[11] A. L. Lloyd and R. M. May, Science 292, 1316 (2001). 
[12] C. Castellano and R. Pastor-Satorras, Phys. Rev. Lett. 96, 038701 (2006).

[13] V. Colizza, R. Pastor-Satorras, and A. Vespignani, Nat. Phys. 3, 276 (2007).

[14] A. Baronchelli, M. Catanzaro, and R. Pastor-Satorras, Phys. Rev. E 78, 016111 (2008).

[15] S. C. Ferreira and M. L. Martins, Phys. Rev. E 76, 036112 (2007).

[16] N. Van Kampen, Stochastic Processes in Physics and Chemistry (Elsevier, Amsterdam, 2007).

[17] I. Hanski and O. E. Gaggiotti, Ecology, Genetics, and Evolution of Metapopulations (Elsevier Academic, Burlington, MA, 2004).

[18] H. Nakao and A. S. Mikhailov, Nat. Phys. 6, 544 (2010).

[19] V. Colizza and A. Vespignani, Phys. Rev. Lett. 99, 148701 (2007).

[20] J. Saldaña, Phys. Rev. E 78, 012902 (2008).

[21] D. Balcan, V. Colizza, B. Gonçalves, H. Hu, J. J. Ramasco, and A. Vespignani, Proc. Natl. Acad. Sci. USA 106, 21484 (2009).

[22] M. Barthélemy, C. Godrèche, and J.-M. Luck, J. Theor. Biol. 267, 554 (2010).

[23] D. Balcan and A. Vespignani, Nat. Phys. 7, 581 (2011).

[24] J. Marro and R. Dickman, Nonequilibrium Phase Transitions in Lattice Models (Cambridge University Press, Cambridge, UK, 1999).

[25] D. J. Watts and S. H. Strogatz, Nature (London) 393, 440 (1998).

[26] R. Pastor-Satorras, A. Vázquez, and A. Vespignani, Phys. Rev. Lett. 87, 258701 (2001).
[27] S. N. Dorogovtsev and J. F. F. Mendes, Adv. Phys. 51, 1079 (2002).

[28] M. Catanzaro, M. Boguñá, and R. Pastor-Satorras, Phys. Rev. E 71, 056104 (2005).

[29] I. Gradshteyn, I. Ryzhik, A. Jeffrey, and D. Zwillinger, Table of Integrals, Series, And Products, 7th ed. (Academic, Burlington, MA, 2007).

[30] C. Castellano and R. Pastor-Satorras, Phys. Rev. Lett. 100, 148701 (2008).

[31] M. Boguñá, C. Castellano, and R. Pastor-Satorras, Phys. Rev. E 79, 036110 (2009).

[32] M. Boguñá, R. Pastor-Satorras, and A. Vespignani, Eur. Phys. J. B 38, 205 (2004).

[33] M. Catanzaro, M. Boguñá, and R. Pastor-Satorras, Phys. Rev. E 71, 027103 (2005).

[34] M. M. de Oliveira and R. Dickman, Phys. Rev. E 71, 016129 (2005).

[35] S. C. Ferreira, R. S. Ferreira, and R. Pastor-Satorras, Phys. Rev. E 83, 066113 (2011).

[36] S. C. Ferreira, R. S. Ferreira, C. Castellano, and R. PastorSatorras, Phys. Rev. E 84, 066102 (2011).

[37] R. S. Sander, S. C. Ferreira, and R. Pastor-Satorras, Phys. Rev. E 87, 022820 (2013).

[38] S. C. Ferreira, C. Castellano, and R. Pastor-Satorras, Phys. Rev. E 86, 041125 (2012).

[39] R. Juhász, G. Ódor, C. Castellano, and M. A. Muñoz, Phys. Rev. E 85, 066125 (2012).

[40] M. A. Muñoz, R. Juhász, C. Castellano, and G. Ódor, Phys. Rev. Lett. 105, 128701 (2010).

[41] D. ben-Avraham and J. Köhler, Phys. Rev. A 45, 8358 (1992). 